\title{
CORRIGENDUM
}

\section{Examining bidirectional relationships between parenting and child maladjustment in youth with autism spectrum disorder: A 9-year longitudinal study - CORRIGENDUM}

\section{LISA M. DIELEMAN, ${ }^{a}$ SARAH S. W. DE PAUW, ${ }^{a}$ BART SOENENS, ${ }^{a}$ WIM BEYERS, ${ }^{a}$ AND PETER PRINZIE $^{b}$ \\ ${ }^{a}$ Ghent University; and ${ }^{b}$ Erasmus University Rotterdam}

doi:10.1017/S0954579416001243, published by Cambridge University Press, 29 December 2016

The direction of the top arrows in Figures $2-4$ in the original online article was incorrect: the arrows should go from T1 to T3. The entire page containing the corrected figures is re-

\section{Reference}

Dieleman, L. M., De Pauw, S. S. W., Soenens, B., Beyers, W., \& Prinzie, P. (2016). Examining bidirectional relationships between parenting and child maladjustment in youth with autism spectrum disorder: A 9-year printed herein. We regret these errors and any problems they may have caused.

longitudinal study. Development and Psychopathology. Advance online publication. doi:10.1017/S0954579416001243. 


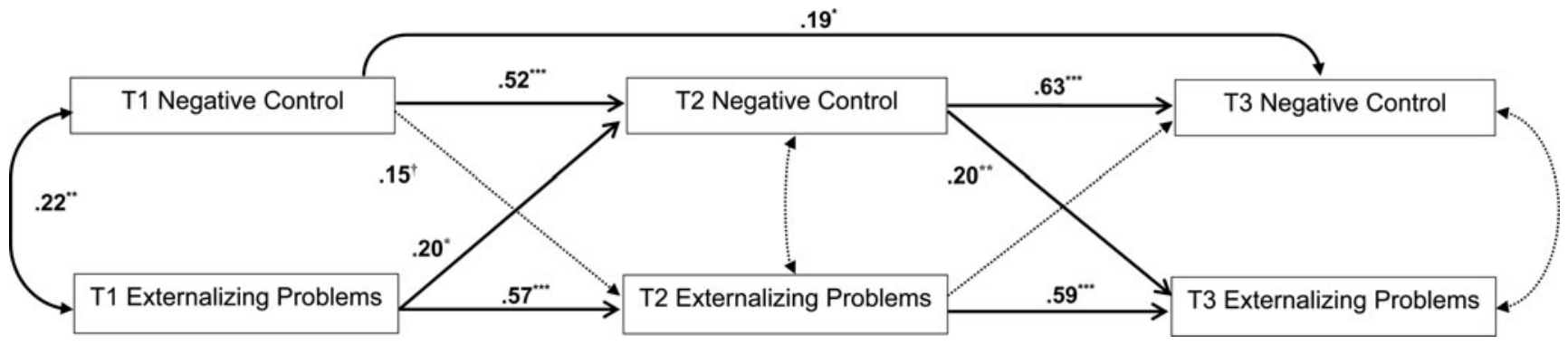

Figure 2. Model of the relationships between negative control and externalizing problems. Standardized path coefficients are presented. Significant relationships are presented in full lines; nonsignificant relationships are presented in dotted lines. T1, Time 1; T2, Time 2; T3, Time 3. $\dagger p<.10 . * p<.05 . * * p<.01 . * * * p<.001$.

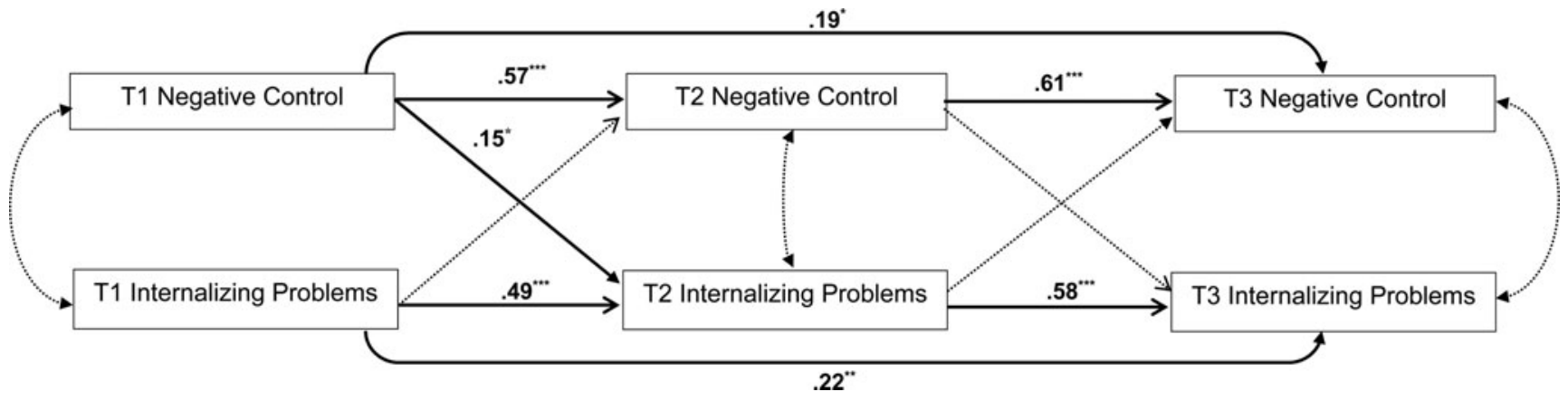

Figure 3. Model of the relationships between negative control and internalizing problems. Standardized path coefficients are presented. Significant relationships are presented in full lines; nonsignificant relationships are presented in dotted lines. T1, Time 1; T2, Time 2; T3, Time 3. $* p<.05 . * * p<.01 . * * * p<.001$.

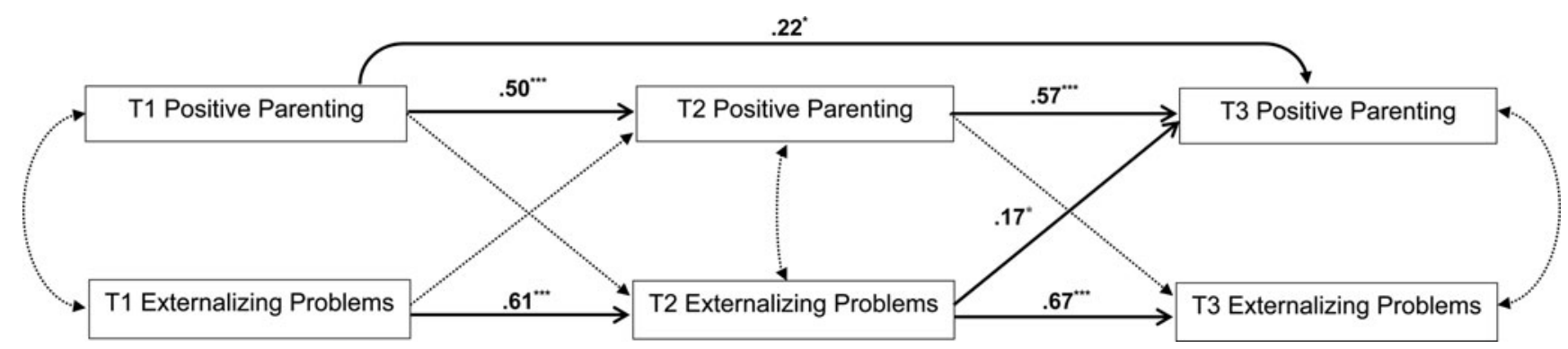

Figure 4. Model of the relationships between positive parenting and externalizing problems. Standardized path coefficients are presented. Significant relationships are presented in full lines; nonsignificant relationships are presented in dotted lines. T1, Time 1; T2, Time 2; T3, Time 3. $* p<.05 . * * p<.01 . * * p<.001$.

The moderating role of gender and autism severity. Our second research question dealt with the moderating role of child gender and autism severity on the longitudinal relations. To this end, multigroup analyses were conducted evaluating whether similar cross-lagged relationships were found across child gender and the two autism severity groups (i.e., those with lower versus higher SCQ-current scores at T1). For both gender and autism severity groups, we compared constrained models (i.e., models in which the parameters for the cross-lagged paths were held constant across groups) to unconstrained models (i.e., models in which the parameters are allowed to vary across groups) evaluating the SatorraBentler scaled chi-square difference test $\left(\operatorname{SBS}^{2} \Delta\right)$.

Gender did not moderate the relationships between negative control and behavioral problems, SBS $\chi^{2} \Delta(8)=$ $14.890, p=.06$ for externalizing, SBS $\chi^{2} \Delta(9)=12.212$, $p=.20$ for internalizing, nor the relationships between positive parenting and behavioral problems, SBS $\chi^{2} \Delta(8)=12.377$, 